\title{
Reforma de los sistemas de salud en América Latina y el Caribe: situación y tendencias
}

\author{
Alberto Infante ${ }^{1}$, Isabel de la Mata ${ }^{2}$ y Daniel López-Acuña ${ }^{1}$
}

RESUMEN A principios de la década de los noventa, casi todos los países de América Latina y el Caribe estaban iniciando, o considerando iniciar, reformas del sector de la salud. El presente trabajo presenta un análisis de la situación de dichas reformas y sus tendencias a finales de la década de los noventa. Para ello los autores han partido de la información contenida en los 20 perfiles de sistemas de servicios de salud concluidos por la Organización Panamericana de la Salud entre agosto de 1998 y octubre de 1999. Siguiendo una metodología empleada con anteriori dad, el análisis se organiza en dos niveles: 1) seguimiento de los procesos (dinámica y conteni dos) y 2) evaluación de los resultados.

En términos de la dinámica de los procesos, el artículo presenta un análisis del contexto y de los actores involucrados en las distintas fases de las reformas: su génesis, diseño y negocia ción, implementación y evaluación. Respecto a los contenidos de las reformas, se describen y analizan los hallazgos organizados en 12 grandes campos temáticos. La evaluación de los re sultados se hace sobre los ocho países que proporcionaron información pertinente y debe consi derarse preliminar. No obstante, el artículo presenta información detallada sobre los resulta dos de las reformas en términos de equidad, efectividad y calidad, eficiencia, sostenibilidad y participación y control sociales. También examina las posibles causas y factores condicionan tes de los resultados observados.

A principios de la década de los noventa, casi todos los países de América Latina y el Caribe habían iniciado, o consideraban iniciar, reformas del sector de la salud (1). Qué se entiende por dichas reformas sigue siendo objeto de debate $(2,3)$. En las Américas, una reunión internacional convocada al efecto en 1995 (4) produjo una definición que se ha venido usando ampliamente desde entonces. ${ }^{3}$ Con todo, el seguimiento de los procesos y la evaluación de los resultados de las reformas del sector de la salud plantean problemas conceptuales y metodológicos que distan de estar resueltos $(5,6)$.
Tras casi dos años de trabajo, en junio de 1998 la División de Desarrollo de Sistemas y Servicios de Salud de la OPS redactó y diseminó unos "Lineamientos metodológicos para la preparación de los perfiles de los sistemas de servicios de salud" de los países de la Región. ${ }^{5}$ Se trata de un documento

\footnotetext{
1 División de Desarrollo de Sistemas y Servicios de Salud, Organización Panamericana de la Salud, Washington, D.C., Estados Unidos de América. Dirección postal: 525 Twenty-third St., N.W., Washington D.C. 20037, Estados Unidos de América. Correo electrónico: infantea@paho.org

2 Subsecretaría de Sanidad y Consumo, Ministerio de Sanidad y Consumo, Madrid, España.

3 La reforma del sector de la salud se definió como "un proceso orientado a introducir cambios sustantivos en las diferentes instancias y funciones del sector con el propósito de aumentar la equidad en sus prestaciones, la eficiencia de su gestión y la efectividad de
}

sus actuaciones y con ello lograr la satisfacción de las necesidades de salud de la población. Se trata de una fase intensificada de transformación de los sistemas de salud realizada durante un período determinado de tiempo y a partir de coyunturas que la justifican y viabilizan". Véase el documento de la Organización Panamericana de la Salud titulado "La cooperación de la Organización Panamericana de la Salud ante los procesos de reforma sectorial" [español e inglés], Washington, D.C., 1997.

4 Véase la sección de metodología de la página electrónica de la cita anterior: http:/ / www.americas. health-sector-reform.org Hay una versión impresa:
Organización Panamericana de la Salud. Línea basal para el seguimiento y evaluación de la reforma sectorial. Washington, D.C.: OPS; 1998. (Documento mimeografiado, español e inglés).

5 Programa de Organización y Gestión de Sistemas y Servicios de Salud, División de Desarrollo de Sistemas y Servicios de Salud, Organización Panamericana de la Salud. Lineamientos para la elaboración de perfiles de los sistemas de servicios de salud en los países de la Región. Washington, D.C. OPS; 1998. (Documento mimeografiado, español e inglés) 
redactado con la mayor objetividad posible, de extensión manejable, y fácil de actualizar, donde se describen y analizan de forma sistemática la estructura y la dinámica del sistema de servicios de salud de cada país, incluidas, cuando es el caso, las reformas del sector de la salud. El índice de capítulos, apartados y epígrafes y el desarrollo de su contenido han sido concebidos para facilitar su uso en los niveles nacional y subnacional, así como la comparación entre países. En los "Lineamientos" se describen, en distintos apartados, los componentes básicos de los perfiles de los sistemas y servicios de salud: 1) contexto político, económico y social; 2) organización general, recursos y funciones; 3 ) seguimiento y evaluación de las reformas.

A continuación se presenta un análisis de la situación de las reformas del sector de la salud y sus tendencias a finales de la década de los noventa, partiendo de la información contenida en el tercer apartado - seguimiento y evaluación de las reformas- de los perfiles concluidos entre agosto de 1998 y octubre de 1999.

\section{EL SEGUIMIENTO Y LA EVALUACIÓN DE LAS REFORMAS}

\section{La dinámica de las reformas}

En los "Lineamientos" se examinan las diferentes fases de los procesos de reforma -génesis, diseño, negociación, ejecución y evaluación-, y se identifican los actores principales del proceso tanto en la sociedad como en el propio sector, tanto nacionales como internacionales.

Según la información que existe, en la mayor parte de los países las reformas del sector de la salud responden a reformas generales del Estado (Perú desde 1995) o de la Constitución (Argentina, Colombia ${ }^{6}$ y Ecuador) y forman parte del proceso de modernización del Estado (Argentina y Chile

\footnotetext{
6 Colombia, Ministerio de Salud. El sector salud en Colombia. Documento preparado para la Reunión Especial sobre Reforma del Sector Salud, Washington, D.C., septiembre de 1995.
}

desde 1994 (7), El Salvador, Jamaica, México (8), Nicaragua, Panamá, Paraguay, República Dominicana, Trinidad y Tabago y Uruguay). A veces toman nombres propios, como "nuevo federalismo" en México o "proceso de perfeccionamiento del Estado" en Cuba. También hay casos, como el del Brasil, en los que la reforma del sector de la salud no forma parte de un proceso de reforma total del Estado, sino de un debate más global en torno a la revisión del propio sistema de salud (9). Y en otros, la reforma empezó a gestarse antes, pero no se puso en marcha hasta que se firmaron los préstamos con los bancos internacionales. En algunos países, la reforma del sector se efectúa aisladamente, como un componente de un plan nacional de salud (Guyana y Honduras), o se produce de manera silenciosa (en Ecuador existe, por ejemplo, pero aún no muestra una clara articulación), o no lleva el nombre de "reforma del sector de la salud" porque consiste solo en iniciativas piloto (Guatemala desde 1996). Y en otras ocasiones no se intenta reformar el sector sanitario en su totalidad, sino llevar a cabo algunos proyectos aislados (Uruguay).

También ha sido frecuente la creación de comisiones de reforma general o unidades específicas para la reforma del sector de la salud. Así, en la República Dominicana se creó una Comisión para la Reforma y Modernización del Estado dependiente de la Presidencia; en el Ecuador, se creó una Comisión Técnica de Reforma dentro del Consejo Nacional de Salud ya existente (10); en El Salvador, una Comisión para la Modernización del Estado; en Nicaragua, el Comité Ejecutivo para la Reforma de la Administración Pública; y en Jamaica, el Comité Interministerial sobre la Reforma Administrativa. La rectoría del proceso inicial de diseño y negociación normalmente ha sido ejercida por el Ministerio de Salud Pública, aunque no siempre, ya que en ocasiones, en función del mecanismo especial de diseño, la ha ejercido el Ministerio de Trabajo y Educación (Colombia) o el de Finanzas (Guatemala).

En algunos casos las instancias parlamentarias han sido el ámbito privilegiado de negociación de la reforma del sector de la salud (Colombia (11) y Paraguay); en otros, comisiones nacionales o grupos de apoyo a la reforma de amplio espectro han cumplido o cumplen esa función (Chile, Ecuador (12), Honduras y Jamaica). En casi todos los casos pueden identificarse dos fases de negociación: una dentro del propio gobierno, y otra entre el gobierno y los distintos actores políticos y sociales. Al inicio, o durante la primera fase, las autoridades nacionales publican documentos para explicitar los problemas, objetivos, estrategias y reformas recomendadas. A partir de ahí el debate público se generaliza.

En muchos casos, los gobiernos han tratado, con distintos resultados, de concertar los objetivos, las estrategias y los ritmos de la reforma del sector de la salud con diferentes actores políticos y sociales relevantes. Algunos países han institucionalizado consejos nacionales (Guatemala y Paraguay), comisiones asesoras o foros nacionales periódicos (Brasil) donde las autoridades sanitarias, los aseguradores, los proveedores, los profesionales y los usuarios discuten los problemas prioritarios y las opciones estratégicas, correspondiéndoles posteriormente a las autoridades adoptar las medidas necesarias para poner en marcha las opciones más adecuadas en cada caso. En ocasiones, esas consultas se han realizado sin que se haya creado un foro específico, como parte del proceso de concertación (Argentina, Guyana, México, Nicaragua, Panamá y República Dominicana). La idea de un "pacto social por la salud" con la participación activa de la sociedad civil ha sido defendida con escaso éxito desde ámbitos académicos (5) y políticos (13) en varios países. La participación de la población en esta fase ha sido escasa y se ha tenido en cuenta sobre todo para la organización de actividades puntuales (Costa Rica, Guatemala, Guyana, Perú).

La ejecución de las reformas del sector de la salud suele ser lenta. Hay países (Brasil desde los años ochenta, Chile y Nicaragua) donde es posible distinguir diversas etapas de la reforma. Como en general las reformas toman varios años, han de ser conducidas por gobiernos de diverso signo político. En 
unos casos, las reformas tuvieron carácter global; implicaron profundas modificaciones (o la sustitución) de la legislación sanitaria básica; tuvieron importantes consecuencias intersectoriales; afectaron a la mayoría de las funciones del sector sanitario; modificaron sustancialmente las relaciones entre los actores públicos y privados existentes y abrieron espacios para la aparición de otros nuevos (Bolivia, Colombia, Paraguay, Puerto Rico (14) y República Dominicana). En otros casos, la reforma del sector de la salud ha sido más limitada en razón de su ámbito de aplicación - los servicios de atención a las personas de uno o varios de los subsistemas públicos del país, como en los casos de Argentina (15) y México-, de la estrategia de implementación desarrollada (Argentina, Ecuador y Perú $(16,17))$, o de la función sectorial afectada: por ejemplo, en ocasiones se ha visto afectada solo la provisión, mediante la adopción de un nuevo modelo de gestión para algunos centros dependientes de los ministerios de salud, instituciones de seguridad social o ambos (Costa Rica, Jamaica, Nicaragua y Panamá). Incluso en este último caso, los debates suelen ser amplios y se tiene conciencia de las posibles repercusiones generales del intento. Sin embargo, los objetivos declarados casi siempre persiguen incrementar la eficiencia y mejorar la calidad de la atención sanitaria, promover la equidad entre distintas regiones y grupos sociales, ampliar la cobertura y reorientar la asignación de recursos. En muchos países, las reformas suelen promover la descentralización como una forma de alcanzar dichos objetivos.

El Banco Interamericano de Desarrollo (BID) ${ }^{7}$ y el Banco Mundial (BM) (18) han sido muy activos en el financiamiento de las reformas del sector de la salud desde finales de los años ochenta y todo indica que lo seguirán siendo en el futuro. Normalmente, la gestión de las acciones financiadas por los bancos internacionales queda a cargo

\footnotetext{
Solo en 1998 el Banco Interamericano de Desarrollo aprobó créditos por un monto de US\$ 119 millones para proyectos de reforma sectorial en Jamaica, Paraguay y la República Dominicana.
}

de unidades técnicas ejecutoras específicas cuya coordinación con los ministerios de salud no siempre ha sido fácil.

La evaluación del desarrollo de las reformas del sector de la salud es desigual y suele verse dificultada por el hecho de que los criterios para llevarla a cabo no se han diseñado (Costa Rica, Chile, Guatemala, Guyana, Honduras y Nicaragua) o se están diseñando aún (República Dominicana). A pesar de ello, algunos países ya han efectuado evaluaciones a raíz de las cuales se han recomendado cambios y adaptaciones $\mathrm{y}$, en ocasiones, la modificación de los procesos (Colombia, Nicaragua, Panamá, Perú y Trinidad y Tabago). En otros casos se han establecido mecanismos para realizar evaluaciones periódicas (Cuba), puntuales (Honduras) o iniciales (Costa Rica). Por último, hay países donde es muy pronto aún para evaluar los resultados del proceso de reforma (Guatemala, Guyana y República Dominicana).

\section{Los contenidos de las reformas}

En este apartado los "Lineamientos" contienen información sobre las estrategias de cambio referidas a los 12 contenidos que se muestran en el cuadro 1.

Con la información disponible, una gran mayoría de los países han realizado modificaciones relevantes del marco jurídico. En dos de ellos se ha reformado la constitución para albergar los cambios implícitos en la reforma del sector de la salud, y en otros 13 se han promulgado nuevas leyes básicas de salud o se han modificado de forma importante las normas existentes. En cuatro países ni se han modificado ni se aspira a modificar los marcos jurídicos, y en un país más se está deliberando aún cómo deben enfocarse dichos cambios. Sin embargo, solo nueve países informan haber incorporado alguna definición de equidad en la nueva normativa, y apenas una minoría (6 de los 20) informa que los cambios jurídicos favorecen un enfoque intersectorial, mientras que otros tres informan lo contrario.

Una gran mayoría de los países garantizan expresamente el derecho a los
CUADRO 1. Contenidos de las reformas del sector de la salud

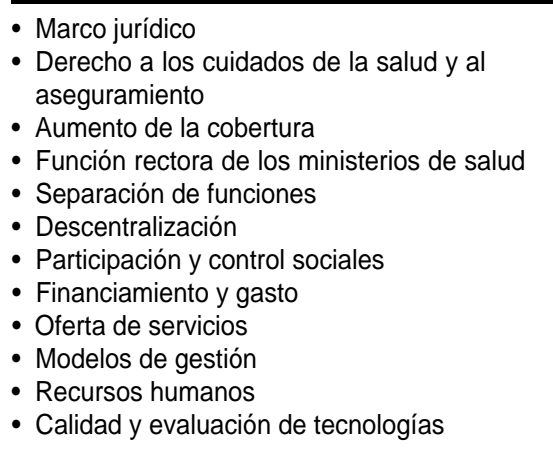

cuidados de la salud, 10 de ellos en sus constituciones, normalmente de forma genérica y sin limitaciones expresas. Sin embargo, solo una minoría ofrecen información que permite inferir que ese derecho es bien conocido por la población o que se desarrollan acciones encaminadas a que lo sea.

También se desarrollan programas para el aumento de la cobertura en 15 de los 19 países que declaran necesitarlos. Casi la mitad de estos programas se basan en aumentar la oferta de servicios básicos de atención primaria a las poblaciones con peor acceso, y a veces en fortalecer programas verticales específicos. En 12 países se han introducido paquetes o conjuntos básicos de prestaciones dirigidos a toda la población o a grupos específicos. Aunque se dispone de poca información sobre su contenido y sobre quién decide su composición, los ministerios de salud parecen tener un papel protagonista y las prestaciones maternoinfantiles suelen ser las más frecuentemente incluidas.

Las reformas del sector de la salud se proponen reforzar la función rectora de los ministerios de salud. En la mayoría de los casos ello ha acarreado cambios en la organización ministerial, si bien solo una minoría de los países (5 de los 20) refieren haber creado nuevas estructuras o instituciones de regulación, dependientes de los ministerios en todos los casos. En la mayoría de los países, la mejora de los sistemas de información parece abordarse en el contexto de las reformas del sector sanitario, pero la información que existe 
sobre el tema no permite sacar conclusiones claras por el momento.

Alguna separación de funciones dentro del sector público se ha producido o se está produciendo en la mayoría de los países; en los ministerios de salud quedan, entre otras, las funciones de regulación, formulación de políticas y coordinación del sector. La información disponible sugiere que varían mucho los efectos de la separación de funciones sobre el financiamiento, que no se dispone de suficiente información sobre cómo afecta al aseguramiento de la salud, y que, al parecer, un buen número de países se han orientado a separar la provisión del resto de las funciones. Y aunque la información es incompleta (9 países de los 20), la impresión es que los mecanismos de rendición de cuentas no se están reforzando o que se refuerzan casi exclusivamente en los aspectos de la auditoría financiera y contable.

En materia de descentralización, solo en cinco casos se advierte una relación entre la descentralización del sector público de la salud y la de otros sectores de la administración pública o del sector social, y en dos de ellos se niega que dicha relación exista. El ámbito privilegiado (13 países de los 20) de la descentralización parece ser el nivel intermedio (regiones, provincias, departamentos o estados) de la administración del Estado, seguido del nivel local (7 países de los 20) y de los propios establecimientos de salud (5 países de los 20). En tres casos se combinan más de un ámbito. Las materias que se transfieren son muy variadas, pero la descentralización suele comenzar por transferir cierta capacidad de administración de los servicios; lo último que se transfiere es la gestión de los recursos humanos, cuya contratación aún se encuentra centralizada en casi todos los países. En cinco países se ofrece información sobre los diversos grados de desconcentración en el seno de cada institución pública.

En la mayoría de los países, impulsar la participación y el control sociales figura entre los objetivos de la reforma del sector de la salud, siendo los niveles gubernamentales intermedios y locales los ámbitos más frecuentes de participación, frente al nivel nacional o al de los establecimientos de salud. Sin embargo, solo siete países ofrecen información sobre la composición de los órganos de participación y su grado de formalización.

En la mayoría de los países se toman medidas para fortalecer los sistemas de información sobre el financiamiento y el gasto en la salud. Sin embargo, en varios casos los sistemas existentes todavía no permiten analizar en conjunto la información procedente de diversas fuentes y es preciso realizar estudios especiales para determinar el gasto, en particular el de carácter privado. La elaboración de cuentas nacionales de salud parece extenderse poco a poco, así como el programa WINSIG de la OPS de apoyo a la gestión. Cambios en la composición del financiamiento se han introducido en ocho países y en otros cuatro se están discutiendo. La mitad de los países están introduciendo cambios en la distribución del gasto público en salud, tanto entre los agentes gastadores como entre los distintos componentes del gasto.

Una gran mayoría de los países está modificando su oferta de servicios públicos, redefiniendo los modelos de atención, modificando la oferta de servicios de primer nivel, e introduciendo o fortaleciendo programas específicos para grupos vulnerables. Sin embargo, solo una minoría parece estar fortaleciendo los sistemas de referencia y contrarreferencia y se dispone de escasa información sobre los cambios realizados en los niveles secundario y terciario y en el sector privado.

La mayoría de los países están introduciendo cambios en los modelos de ges tión y en las relaciones entre los diferentes actores del sector público. Muchos ya tenían, o están estableciendo, alguna forma de compra y venta de servicios a terceros, y la mitad de los países están introduciendo compromisos o acuerdos de gestión entre los financiadores y los proveedores dentro del subsistema público. Muy pocos han introducido modalidades de autogestión en los establecimientos públicos de salud (aunque ello está en discusión en algunos países), dos han entregado a la gestión privada algún servicio público, y solo uno ha privatizado parcialmente los servicios.

Una minoría de los países informa estar introduciendo cambios en la formación y capacitación de los recursos humanos del sector como consecuencia de las reformas sectoriales; cinco países informan estar discutiéndolos. Se observan cambios en la planificación y la gestión de los recursos humanos por los principales empleadores públicos en 11 de los 20 países, pero solo en 7 de ellos muestran suficiente regularidad y dirección. Sin embargo, solo en ocho países existían o se han introducido incentivos económicos para el desempeño (aunque otros cuatro los tienen previstos); únicamente cuatro países han introducido modificaciones en la práctica profesional orientadas a fomentar enfoques multidisciplinarios; $y$ solo cinco han introducido la recertificación de los profesionales de la salud. Se dispone de muy poca información sobre el comportamiento del sector privado. Por último, solo seis países informan una participación fructífera de los trabajadores y profesionales del sector en el proceso de reforma sectorial.

Existen instancias, programas o ambos para fomentar la calidad técnica en la mitad de los países, pero solo cuatro refieren experiencias concretas que hayan fomentado la calidad percibida. Mecanismos o procedimientos para la acreditación de establecimientos y servicios de salud parecen estar funcionando solamente en unos cuantos países (5 de 20, aunque otros tres están considerando su introducción). Por último, solo una minoría de los países han introducido procedimientos para la gestión y la evaluación de las tecnologías de salud.

\section{La evaluación de los resultados de las reformas}

El apartado sobre la "Evaluación de los resultados" se diseñó para determinar hasta qué punto las reformas del sector de la salud pueden estar contribuyendo a mejorar los niveles de equidad, efectividad y calidad, eficiencia, sostenibilidad y participación y control social de los sistemas y servicios de sa- 
lud, ya que estos fueron los cinco principios rectores de las reformas identificados por los Gobiernos americanos durante la reunión especial sobre la reforma del sector de la salud de 1995.

Lo que sigue se basa en el análisis de los 17 perfiles concluidos hasta marzo de 1999. Aunque todos incluían información relevante para el seguimiento del proceso de reforma sectorial (es decir, de la dinámica y de los contenidos de las reformas), solo ocho contenían información sobre los resultados de las mismas. En lo que respecta a los nueve perfiles restantes, la ausencia de información se consideró justificada por el escaso tiempo transcurrido desde el inicio de la reforma (2 años o menos) en seis de ellos, y no justificada en tres. ${ }^{8}$

Equidad. Los "Lineamientos" buscan conocer hasta qué punto hay pruebas de que las reformas del sector de la salud están influyendo sobre 18 indicadores cuantitativos y cualitativos referidos a cobertura, distribución, acceso y uso de recursos.

La información obtenida sugiere que, en términos de equidad, las reformas del sector pueden haber contribuido o estar contribuyendo a reducir las disparidades, al menos en la cobertura de una serie de programas y servicios básicos, en la mitad de los países analizados. También sugiere que solo en una minoría de los países existen pruebas de que las reformas están contribuyendo a reducir las disparidades en la distribución de recursos. La calidad de la información

\footnotetext{
8 El análisis de un cuestionario independiente dirigido a los funcionarios de la Organización Panamericana de la Salud que actuaron como puntos focales nacionales para la elaboración de los "perfiles" en los países reveló que las razones más comúnmente aducidas para explicar las ausencias de información sobre los resultados de las reformas sectoriales tienen que ver con que: 1) las propuestas iniciales de reforma del sector no suelen incluir mecanismos para evaluar el impacto de las reformas ni para vigilar el desarrollo del propio proceso de reforma, ni plazos concretos para lograr los objetivos propuestos; 2) la selección de variables e indicadores para evaluar los resultados es técnicamente complicada y las atribuciones de causalidad son difíciles; 3) la información disponible en los países a menudo es incompleta, se halla dispersa, resulta poco fiable o es inoportuna, y 4) los actores principales (entre ellos las autoridades de salud) no siempre muestran suficiente interés en evaluar los resultados.
}

sobre la equidad en el acceso y uso de los recursos por ahora no permite sacar conclusiones sobre estos dos apartados.

Efectividad y calidad. Los "Lineamientos" buscan conocer hasta qué punto hay pruebas de que las reformas del sector de la salud están influyendo sobre 19 indicadores cuantitativos y cualitativos referidos a efectividad global, calidad técnica y calidad percibida. En términos de efectividad y calidad, la información sugiere que: 1) solo en una minoría de los países (dos de los ocho) las reformas han contribuido a mejorar la efectividad global del sistema; 2) solo en una exigua minoría de los países las reformas están contribuyendo a mejorar la calidad técnica y, 3) solo en una minoría de los países las reformas han contribuido a mejorar la calidad percibida.

Eficiencia. Los "Lineamientos" buscan conocer hasta qué punto hay pruebas de que las reformas del sector sanitario están influyendo sobre 19 indicadores cuantitativos y cualitativos referidos a eficiencia en la asignación y gestión de los recursos. En términos de eficiencia en la asignación de recursos, la información sugiere que: 1) en la mitad de los países analizados las reformas pueden estar contribuyendo a la introducción de mecanismos más eficientes de asignación de recursos; 2) solo en una exigua minoría de los países han contribuido a mejorar los indicadores de la eficiencia global del sistema $y, 3$ ) solo en una minoría de los países han contribuido a una reasignación de los recursos para acciones intersectoriales y programas de prevención de enfermedades y trastornos de alta prevalencia.

En términos de eficiencia en la gestión de recursos, la información sugiere que: 1) en una mayoría de los países las reformas sectoriales están contribuyendo a la introducción de "compromisos de gestión"; 2) solo en una minoría de los países han contribuido a la aplicación de medidas de actividad estandarizadas, a la mejora de indicadores básicos de rendimiento hospitalario, al empleo autónomo de nuevas fuentes de ingreso por los establecimientos sanitarios públicos o a la preparación de presupuestos basados en criterios de actividad.

Sostenibilidad. Los "Lineamientos" buscan conocer hasta qué punto hay pruebas de que las reformas del sector de la salud están influyendo sobre seis indicadores cualitativos referidos a la legitimación y sostenibilidad financiera de las principales instituciones públicas. La información al respecto sugiere que, por ahora, las reformas han contribuido a: 1) mejorar la información desagregada sobre el gasto en salud, aunque no hay pruebas de que hayan contribuido a mejorar la capacidad de los países para construir tendencias y elaborar escenarios de gasto; 2) mejorar en la mitad de los países, como mínimo, la capacidad para ajustar ingresos y gastos en el subsistema público y para cobrar a terceros obligados a pagar; 3) mejorar en una minoría de los países la legitimación de las principales instituciones públicas prestadoras, la sostenibilidad a mediano plazo de los programas y servicios, o la capacidad de gestión presente de los préstamos externos y su eventual sustitución futura.

Participación y control sociales. En este caso los "Lineamientos" buscan conocer si las reformas han aumentado los espacios de participación y control social, tanto de la población en general como de algunos grupos específicos. Parece haber bastantes pruebas de que las reformas están contribuyendo a aumentar los espacios de participación social en los distintos niveles del sistema en la gran mayoría de los países.

\section{DISCUSIÓN}

En términos de la dinámica de los pro cesos, cabe destacar que en sus orígenes las reformas del sector de la salud se vieron muy condicionadas por los procesos de reforma del Estado, el predominio de las consideraciones pura- 
mente financieras y la escasa influencia de los ministerios de salud en su diseño. Sin embargo, desde mediados de la década de los noventa se ha avanzado en el reconocimiento de su complejidad y especificidad, en el reequilibrio entre actores nacionales e internacionales, y en cierta recuperación del liderazgo de las autoridades nacionales de salud.

Si se aplica rigurosamente la definición inicial, no todos los cambios introducidos en el sector de la salud en los países podrían llamarse "reformas". En algunos casos los cambios son sustantivos y planificados pero no se denominan de ese modo; en otros, se modifica la totalidad de las funciones de una de las instituciones públicas importantes, pero no la de las restantes. En algunos países, como Brasil, Chile, Cuba, Ecuador y Trinidad y Tabago, es posible caracterizar, a lo largo de la última década, dos o tres períodos de reforma sectorial; en otros aún no es posible detectar ningún proyecto reformista.

A mediados de 1999, la mayoría de los países de América Latina y el Caribe se encontraban en algún punto comprendido entre el diseño y las primeras fases de la implementación de las reformas del sector de la salud. En una minoría creciente la implementación se encontraba avanzada y en unos pocos países ya se empezaba a hablar de una "segunda" o incluso una "tercera" generación de reformas sectoriales.

En casi todos los países, el papel de los organismos de cooperación financiera es cada vez más importante. Sin embargo, las autoridades de salud no siempre parecen haber desempeñado el papel que les correspondería en la formulación de las políticas nacionales de financiamiento y gasto sectorial o en la definición de las áreas prioritarias para la concesión de los créditos internacionales.

La evaluación del desarrollo de los procesos de reforma sectorial es incipiente y desigual. En la mayoría de los países no se realiza de manera sistemática y en los pocos donde ello se cumple, rara vez se usa como insumo importante para rediseñar el contenido y las estrategias de implementación de las reformas.
En lo que respecta a los contenidos, sorprende que en la mitad de los países no se mencione el objetivo teórico ulterior de cualquier reforma, que es mejorar la equidad. Sin embargo, en la mayor parte de los países se desarrollan programas de aumento de la cober tura, dirigidos a incrementar la oferta básica de servicios a las poblaciones más desfavorecidas, lo cual contribuiría, teóricamente, a mejorar la equidad. A menudo se perciben ciertas confusiones conceptuales: entre aumentar la cobertura y ofrecer paquetes básicos de prestaciones; entre la descentralización y la desconcentración; y entre la privatización y los nuevos modelos de gestión. Asimismo se detectan dos incongruencias notables: entre el reconocimiento, por un lado, de la necesidad de mejorar los sistemas de información para seguir los procesos de reforma y evaluar los cambios conseguidos y, por otro, el escaso esfuerzo que aún se dedica a tal mejora; y entre el reconocimiento de la importancia de los recursos humanos y su escasa implicación en los procesos de reforma del sector de la salud.

En lo referente a los resultados de las reformas, sorprenden los obtenidos en el campo de la equidad de la cobertura, dado que casi todos los programas de reforma aspiran a aumentar la cobertura regular de los servicios básicos a los cuales se refieren los indicadores seleccionados. Está por ver cómo se comporta esta variable a corto plazo, a medida que aumenta el número de perfiles completados, y a mediano plazo, al avanzar los procesos de reforma del sector sanitario y mejorar la calidad de la información disponible mediante las sucesivas revisiones de los perfiles.

El resultado en cuanto a la equidad de la distribución de los recursos puede parecer desalentador pero no es sorprendente, pues la mayor parte de los indicadores usados para evaluar esta variable son más difíciles de modificar en el corto plazo y, para evaluar sus resultados, se requieren series temporales consolidadas que aún son escasas en la mayoría de los países.

Llaman la atención los resultados referidos a la efectividad global porque, de los indicadores incluidos para evaluar esta variable, por lo menos cuatro son de uso común y son comunicados con regularidad por los países. Estos indicadores se consideran, además, sensibles en el corto plazo a las modificaciones de las políticas de salud y se refieren a asuntos que suelen tener prioridad entre las políticas de reforma sectorial. Por el momento no es posible descartar la posible presencia de un sesgo de selección que pueda desaparacer a medida que aumenta la lista de países con perfiles concluidos, o de un artefacto inducido por el propio instrumento de evaluación y que se corrija en la próxima revisión de los "Lineamientos". Por otro lado, dado que para completar el análisis se usó también la información sobre el tema contenida en el segundo apartado de los perfiles -organización general, recursos y funciones-, los pobres resultados de las reformas en ambos apartados parecen, por el momento, bastante concordantes.

Los resultados en términos de eficiencia concuerdan con el énfasis que han puesto muchas de las reformas en curso en introducir "desde arriba" los "compromisos de gestión" como un modo de mejorar la eficiencia en la asignación de recursos desde los financiadores públicos hacia los proveedores. También concuerdan con el hecho de que el efecto de las reformas sobre el manejo de medidas estandarizadas de actividad y otros instrumentos destinados a mejorar la gestión (por ejemplo, el empleo sistemático de indicadores básicos de rendimiento, presupuestos basados en criterios de actividad, o el empleo autónomo de nuevas fuentes de ingreso) dentro de los establecimientos (particularmente en los hospitales) es más lento o menos evidente. $Y$ también es compatible con el temor expresado por diversos observadores, antes (19) y ahora (20), ${ }^{9}$ de que las acciones intersectoriales de alta externalidad y los programas de prevención ocupen un lugar

\footnotetext{
9 Alleyne G. Health reform. The World Bank's Human Development Week, Washington, D.C., 25 a 27 de marzo, 1997. (Documento mimeografiado)
} 
secundario en las políticas de reforma del sector de la salud.

Los resultados observados en el campo de la sostenibilidad parecen concordar con el énfasis en el control del gasto y en la recuperación de costos en la mayoría de las reformas sectoriales, pero ponen de manifiesto tres de las principales debilidades estratégicas del subsector público en el actual contexto de reforma: la baja legitimación, aún no resuelta; la precaria sostenibilidad de los nuevos programas, y la creciente dependencia de la financiación externa.

Los resultados en cuanto a participación y control sociales parecen favorables y, en una primera aproximación, pareciera que pueden contribuir a paliar algunos de los factores, señala- dos anteriormente, que limitan la sostenibilidad. Sin embargo, dadas la gran variedad de modelos y experiencias en curso $(21)^{10,11}$ y su compleja vincula- ción en muchos países con los procesos de descentralización, ${ }^{12,13}$ el tema exige un análisis pormenorizado que excede los límites del presente trabajo.
10 Cortez R, Phumpu P. The delivery of health services in shared community-state administration centers: the case of Peru. Informe de política del International Development Research Center y del Banco Interamericano de Desarrollo que se distribuyó en la reunión "Social policy reforms in Latin America", celebrada en Washington, D.C., en mayo de 1999. El documento puede pedirse al Centro de Investigación de la Universidad del Pacífico, Lima, Perú; dirección electrónica: rcortez@up. edu.pe

11 Yepes FJ, Sánchez LE, Cantor B. Decentralization of health at the municipal level: case and control studies. Informe de política del International Development Research Center y del Banco Interamericano de Desarrollo que se distribuyó en la reunión "Social policy reforms in Latin America", celebrada en Washington, D.C., en mayo de 1999. Asociación Co- lombiana de la Salud, Bogotá, Colombia. Dirección electrónica: assalud@colciencias.gov.co

12 Organización Panamericana de la Salud. La descentralización, los sistemas de salud y los procesos de reforma del sector. Informe final de la reunión celebrada en Valdivia, Chile, del 17 al 20 de marzo de 1997. Washington, D.C.: OPS/OMS; 1997. (Serie Organización y Gestión de Sistemas y Servicios de Salud, documento mimeografiado, español e inglés)

13 Sterman L, coord. The decentralization of the Brazilian health system. Informe de política del International Development Research Center y del Banco Interamericano de Desarrollo que se distribuyó en la reunión "Social policy reforms in Latin America", celebrada en Washington, D.C., en mayo de 1999. El documento puede pedirse al Instituto da Saúde, Secretaria de Estado da Saúde de Sao Paulo, Brasil; dirección electrónica: nisis@isaude.sp.gov.br

\section{REFERENCIAS}

1. Pan American Health Organization, Executive Committee of the Directing Council. Progress of activities in health sector reform. Presentado en la 26. ${ }^{\text {a }}$ Reunión del Subcomité de Planificación y Programación, Washington, D.C., 25 a 27 de marzo de 1996. (SPP27/7 [inglés]).

2. Berman P. Health sector reform: making health development sustainable. Health Policy 1995;32:13-28.

3. Saltman RB, Figueras J. Reforma sanitaria en Europa: análisis de las estrategias actuales. Ginebra y Madrid: Organización Mundial de la Salud (Oficina de Europa) y Ministerio de Sanidad y Consumo; 1997.

4. Banco Interamericano de Desarrollo, Banco Internacional de Reconstrucción y Fomento, Comisión Económica para América Latina y el Caribe, Organización de los Estados Americanos, Organización Panamericana de la Salud, Fondo de Población de las Naciones Unidas, Fondo de las Naciones Unidas para la Infancia, Agencia de los Estados Unidos para el Desarrollo Internacional. Reunión Especial sobre Reforma del Sector Salud. Informe de la Reunión, 29 a 30 de septiembre de 1995, Washington D.C.; 1996.

5. Frenk J. Comprehensive policy analysis for health system reform. Health Policy 1995; 32:257-277.

6. Infante A. La elaboración de perfiles de sistemas de servicios de salud y el seguimiento y evaluación de las reformas del sector como ins- trumentos para el desarrollo de políticas sanitarias en las Américas. En: Ministerio de Sanidad y Consumo, Organización Mundial de la Salud y Agencia Española de Cooperación Internacional. Mesa redonda sobre investigación en salud pública y desarrollo, Jornadas de Cooperación Sanitaria, 29 de junio al 1 de julio, Madrid, 1998.

7. Jiménez de la Jara J, Bossert T. Chile's health sector reform: lessons from four reform periods. Health Policy 1995; 32:155-166.

8. México, Poder Ejecutivo Federal. Programa de reforma del sector salud, 1995-2000. México, D.F.: PEF; 1995.

9. De Siquiera BP. Reformas de los sistemas de salud: el caso de Brasil. En: Perú, Ministerio de Salud. Seminario Internacional sobre Reforma del Sector Salud. Lima: Ministerio de Salud; 1997. pp. 55-60.

10. Ecuador, Consejo Nacional de Salud. Hacia la reforma del sector salud. Quito: Culturales; 1995.

11. Ministerio de Salud de Colombia y Organización Panamericana de la Salud. La reforma del sector salud en Colombia. Santa Fe de Bogotá: MS, OPS; 1995.

12. Palacio A. La experiencia de la Comisión Interagencial (CIAR) de Apoyo a la Reforma en el Ecuador. En: Organización Panamericana de la Salud. Reforma del sector salud en el Área Andina. Quito: OPS; 1996. pp.253-256.
13. Echevarría R. El proceso de reforma del sector salud en Ecuador, 1992-1997: aportes para el debate. Quito: CEPAR; 1997.

14. Organización Panamericana de la Salud. Reforma del sector salud: el caso de Puerto Rico. Washington, D.C.: OPS; 1998.

15. González García G. La reforma del sector salud en la Argentina. En: Memoria del $1^{\mathrm{er}}$ seminario sobre los procesos de reforma de la seguridad social en materia de salud. México, D.F.: Confererencia Interamericana de Seguridad Social, Secretaría General; 1998. (Serie Estudios No. 42).

16. Organización Panamericana de la Salud. Las condiciones de salud en las Américas, edición de 1998, vol. II. Washington D.C.: OPS; 1998.

17. Aguinaga A. Avances en el proceso de reforma en el sector salud. En: Reforma del sector salud en el Área Andina. Quito: OPS; 1996.

18. Inter-American Development Bank. Annual report, 1997. Washington, D.C.: IADB; 1998.

19. Banco Mundial. Estrategia sectorial: salud, nutrición y población. Washington, D.C.: BM; 1997.

20. Tejada de Rivero D. The financing of health: conditions for effectiveness and equity. PAHO Bull 1998; 22(4): 447-455.

21. Tarimo E. Essential health service packages: uses, abuse and future directions: current concerns. Geneva: World Health Organization; 1997. (ARA Paper No. 15). 
ABSTRACT In the early 1990s, most Latin American and Caribbean countries were beginning, or planning to begin, health sector reform processes. This paper presents the status and trends of health sector reform at the end of the 1990s. The authors relied on information

Health sector reform in Latin America and the Caribbean: situation and trends in 20 health system and services profiles completed by the Pan American Health Organization between August 1998 and October 1999. The analysis, which follows a methodology that had been applied earlier, was organized on two different levels: (1) monitoring the reform processes (dynamics and content) and (2) evaluating their outcomes.

In looking at the dynamics of the reform processes, the article examines the context in which they take place and the actors involved in their different phases: inception, design and negotiation, implementation, and evaluation. The description and analysis of the contents of health sector reform initiatives are organized into 12 broad thematic areas. Outcomes evaluation was only possible in the eight countries that provided enough pertinent information, and should be viewed as preliminary. Nevertheless, the article does present detailed information on the outcomes of health sector reform in terms of equity, effectiveness and quality, efficiency, sustainability, and societal participation and control. The article also discusses the potential causes and determining factors for the observed outcomes. 